\title{
EXPERIMENTAL INVESTIGATION OF THE LOSS CHARACTERISTICS OF SUPERCONDUCTORS FOR AC POWER APPLICATIONS*
}

Frank van Overbeeke, Klaas oordt and Louis J.M. van de Klundert

Twente University of Technology, Department of Applied Physics, P.0.B. 217, 7500 AE ENSCHEDE, The Netherlands

\section{Summary}

Recent studies $^{1,2}$ confirm the economical feasibility of the application of superconductors in AC power apparatus. The low-loss conductor which has been proposed still only exists in theory. Simple calculations show that the economic criterion described by Ogasawara ${ }^{3}$ and Hlásnik ${ }^{1}$ is inaccurate because of an oversimplified hysteresis loss formula. A necessary modification shows that stricter demands will have to be made. As production of ultrafine filaments is a big technological problem, final diameter reduction by rolling instead of drawing is proposed. The expected better performance of a rolled composite has been verified experimentaliy in test coils as well as in transformér geometry. Expeximental set-up and results are presented.

\section{Introduction}

The application of superconducting windings in $\mathrm{AC}$ power apparatus has been object of study for over 20 years. It is only for the last few years however that this option has become economically interesting, because of the increased cost of energy, the increased efficiency and reliability of cryogenic apparatus and the developments concerning low-loss AC conductors. The extremely low AC loss level of the conductor proposed by Riemersma et al. in their design of a 1000 MVA transformer ${ }^{2}$, is in any case an upper limit of the loss acceptable. With higher losses the economical benefit would decrease or even become negative.

We must be quite aware of the way in which the $A C$ losses are calculated. Firstly, we have to distinguish between eddy current losses and superconductor hysteresis losses, which is quite usual. We believe that in a carefully designed AC-composite the latter will dominate the former. Secondly, the hysteresis losses consist of a magnetization contribution of the individual filaments (due to the field of the coil system in which the conductor is located) and of a self-field contribution (due to the current in the wire itself). These two contributions interfere with each other, and generally the total loss is less than the sum of both of them, but not less than each of them apart ${ }^{4}$. The selffield term may, for every thin filaments, well exceed the magnetization term, especially when, thirdly, a dependence $J_{C}=J_{C}(B)$ is introduced. This can be shown numerically as well as experimentally. Surprisingly the self-field term is neglected in, for instance, economic criteria described by Ogasawara ${ }^{3}$ and Hlásnik ${ }^{1}$.

\footnotetext{
* Work supported by the Netherlands Foundation for Fundamental Research on Matter (FOM)
}

\section{Loss calculation}

Let us take, for example, Ogasawara's hysteresis loss formula (11):

$$
\begin{gathered}
P_{h}=\frac{4 \omega d \lambda J_{c} \hat{B}}{3 \pi^{2}\left(1+\omega^{2} \tau^{2}\right)^{\frac{3}{2}}} \\
\text { or, with }\left(\omega^{2} \tau^{2}\right)<1 \\
P_{h}=\frac{4 \omega}{3 \pi^{2}} \lambda J_{c} d \hat{B}
\end{gathered}
$$

in which $\mathrm{Ph}_{\mathrm{h}}$ is the loss per unit volume $\left[\mathrm{W} / \mathrm{m}^{2}\right]$ of the wire, $\lambda$ is the fraction of the wire that is superconducting, $J_{C}$ is the critical current density $\left[\mathrm{A} / \mathrm{m}^{2}\right], \hat{B}$ the amplitude of the $A C$ magnetic field, $\omega=2 \pi f$ the frequency of the $\mathrm{AC}$ field, $a$ the filament diameter and $\tau$ the time constant of the composite. we have a self-field term

$$
\begin{aligned}
P_{S} & =\frac{1}{\pi R^{2}} \frac{\mu_{0} f}{6 \pi} \cdot \frac{\hat{I}^{3}}{I_{C}} \\
& =\frac{\mu_{0} \omega}{12 \pi^{2}} \cdot \pi R^{2}\left(\lambda J_{C}\right)^{2} \cdot \frac{1}{\eta^{3}}
\end{aligned}
$$

where $P_{S}$ is the self-field loss per unit volume, $R$ is the wire radius, $\hat{I}$ is the amplitude of the $A C$ current and $\eta$ is the "safety margin" $I_{C} / \hat{I}$ which, for $\mathrm{d}=0.5 \mu \mathrm{m}, \hat{\mathrm{B}}=0.3 \mathrm{I}$ and $\mathrm{\eta}=1.5$ renders $\mathrm{P}_{\mathrm{h}}=\mathrm{p}_{\mathrm{s}}$ for

$$
\pi R^{2} \lambda J_{C}=6.5 \mathrm{~A}
$$

This value of $6.5 \mathrm{~A}$ is much smaller than the maximum currents usually encountered in $A C$ conductors. For a wire with only a ring of filaments between the radii $r=r_{0}$ and $r=R$, we obtain

$$
P_{S}=\left(1-\frac{r_{0}^{2}}{R^{2}}\right) \frac{\mu_{0} \omega}{12 \pi^{2} R^{2}} \cdot \frac{\hat{I}^{3}}{I_{C}}
$$

which offers some reduction in $\mathrm{P}_{\mathbf{S}}$, when we choose $\hat{\mathrm{I}}$ and $I_{C}$ to be the same as above. Still it can be seen that, unless the safety margin $\eta$ is chosen considerably higher, the self-field loss will at least be of the same order of magnitude as the magnetization loss.

We can conclude that a low magnetization loss. is attained with a small filament diameter, and that a low self-field loss necessitates a small critical current or a high safety margin, and a high $r_{0} / R$ ratio. The dependence $J_{C}=J_{C}(B)$ must undoubtedly be taken into account as well. It can be shown numerically that this does not affect the above conclusions qualitatively, but that is does so very much quantitatively.

Up to now most effort has been spent on the reduction of the filament diameter, which will also be the topic in the rest of this paper. 
Reduction of the magnetization loss of a multifilamentary wire

We recall here the mentioned formula for the magnetization loss of a multifilamentary composite with thin filaments and $J_{c}=$ constant:

$$
P_{h}=\frac{4 \omega}{3 \pi^{2}} \lambda J_{c} d \hat{B}
$$

The diameter $d$ refers to that of a cylindrical filament, but an almost equal formula applies to a slab in a parallel field, of which $a$ is the thickness perpendicular to the field ${ }^{5}$ :

$$
P_{h}=\frac{\omega}{2 \pi} \lambda J_{C} d \hat{B}
$$

Assume we have a multifilamentary wire with given wire and filament diameters. In order to reduce its AC field losses by a factor 2 the wire can be drawn to half its diameter. Afterwards this wire will have to be cut into four parts which must again be parallelled to obtain a wire of the same length and current-carrying capacity.

Should this wire be used in a geometry of, for instance, a transformer where the field direction is well-known and time-independent, another solution could be worthwhile: By rolling this same wire to about 0.4 times its diameter, a large part of the filaments is also flattened and will thus have a magnetization behaviour comparable to that of slabs. This rolledwire has about the same length and current-carrying capacity as its round original. In this way we have produced a twisted multifilamentary tape.

The question is whether a multifilamentary composite can withstand this kind of "bad treatment". For composites with NbTi filaments in a copper-nickel matrix this question can be answered affirmatively. Photomicxographs have been made of every rolled sample such as the one in figs. 6 and 7 , showing that:

- filaments at the edges of the "tape" have undergone a negligible deformation

- filaments in the center of the tape have undergone maximum deformation, but seem to be intact

- a layer of matrix material remains between filaments (see fig. 7).

Furthermore, etching of the matrix has proved that the filaments have not been broken. Breaking of the filaments is a major problem when drawing and twisting wires with thin filaments.

Several types of commercially available multifilamentary high-resistivity matrix composites have been treated in this way. Measurements have been performed to establish AC losses and critical currents in comparison with the respective original round wires. In all cases a diameter reduction of a factor 3 in one step appeared to be possible. This factor was not limited by the wire, but by the quality of the roll surface.

Table I

\begin{tabular}{|c|c|c|c|c|}
\hline Manufacturer & $\begin{array}{l}\text { No. of } \\
\text { filaments }\end{array}$ & $\begin{array}{l}\text { Filament } \\
\text { diameter }\end{array}$ & $\begin{array}{l}\text { Wire } \\
\text { diameter }\end{array}$ & $\begin{array}{l}\text { After } \\
\text { rolling }\end{array}$ \\
\hline IMI & 402 & $10 \mu \mathrm{m}$ & $0.25 \mathrm{~mm}$ & $0.06 \mathrm{~mm}$ \\
\hline MCA & 574 & $8.5 \mu \mathrm{m}$ & $0.29 \mathrm{~mm}$ & $0.08 \mathrm{~mm}$ \\
\hline MCA & 114 & $13 \mu \mathrm{m}$ & $0.20 \mathrm{~mm}$ & $0.05 \mathrm{~mm}$ \\
\hline
\end{tabular}

\section{Experiments}

The test coils consisted of $4 \times 35$ or $4 \times 40$ windings, non-inductively wound on a coil former with a diameter of $25 \mathrm{~mm}$ (fig. 1). Wire spacing has been chosen to be the same for a rolled wire and its round counterpart. Maximum field between layers 2 and 3 was about $2.2 \mathrm{mT} / \mathrm{A}$. Table I sunmarizes the investigated wires and reduction factors. Figure 2 shows the measured loss of the different test coils as a function of the AC current amplitude, scaled on the loss of each round wire at $\hat{\mathrm{I}}=50 \mathrm{~A}$.

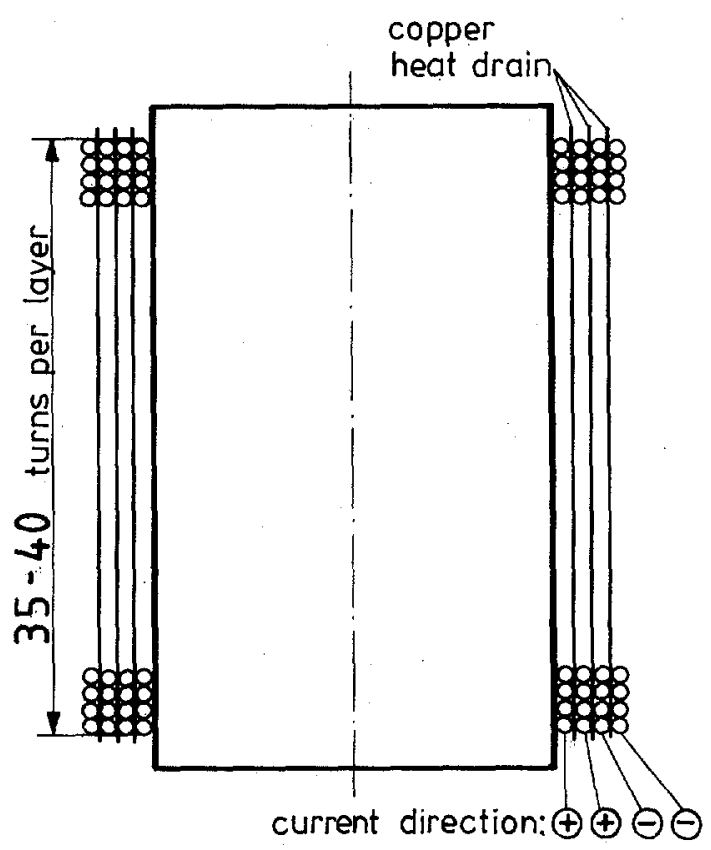

Fig. 1. Test coil for loss measurements.

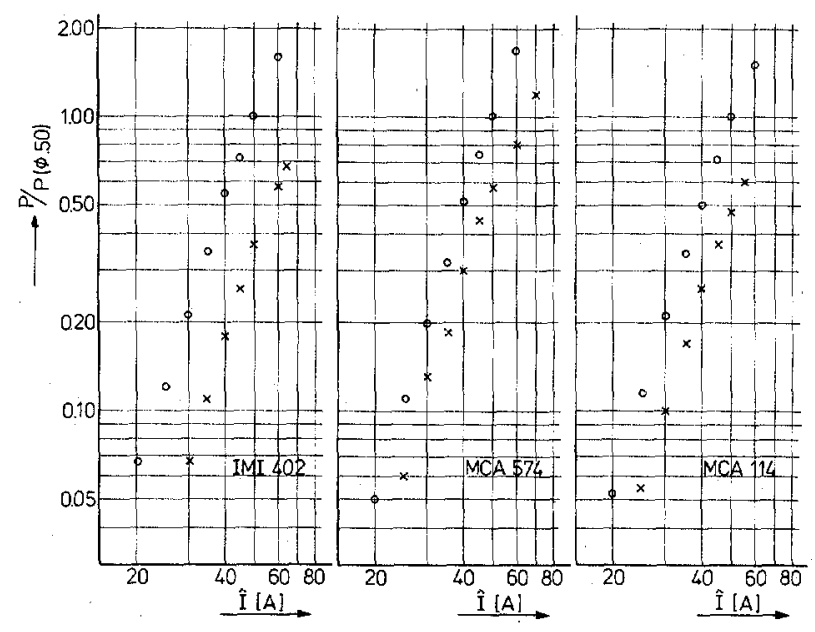

Fig. 2. Losses for three different conductors: original round wire (0) and rolled wire(x). In each graph the loss of the round wire for $\overline{\mathrm{I}}=50 \mathrm{~A}$ is chosen as unity. 
One of these wires has been chosen for more extensive studies, namely the IMI 402 filaments wire. In figure 3 maximum currents of the test coils of this wire have been depicted as a function of frequency, showing a considerable increase after rolling. It must be emphasized that the points shown here refer to the lowest observed quenching current. At frequencies below $20 \mathrm{~Hz}$ measurements on the round wire were possible at much higher current values, accepting a periodical quenching of the coil. The higher maximum curxent of the rolled wire therefore has little to do with a change in $J_{C}$, but with improved stability due to a larger surface and consequently better heat transfer to the surroundings.

This type of rolled conductor has also been used to. construct a superconducting transformer. The transformer consists of an iron transformer core, for convenience also submersed in the licuid helium, and a primary and a secondary coil of 240 turns each. Both coils have been protected with auxiliary coils in the way suggested by authors such as Riemersma and Murphy ${ }^{2}, 6$. The behavioux of this protection system will be reported elsewhere ${ }^{7}$.

Fig. 5 shows the measured loss per cycle of this coil system, when connected according to the scheme of fig. 4. This way of connecting makes operation at minimum core loss possible. Losses were measured calorimetrically as well as electrically, the latter by means of analogue compensation circuitry and a process computer. The difference between electrical and calorimetric measurement results is accounted for by the dissipation in the transformer core, of which the off-gas is not led into the mass flow meter.

The third curve represents the loss values predicted from the (electrically measured) losses of the smoll. test coils. These have been scaled assuming a linear relation between $\mathrm{P}$ and $\hat{I}$ for $\hat{I}>50 A$, and further assuming that the loss is only caused by the AC fiela and not by the $A C$ current in the wire itself. This simple approximation appears to hold quite well.

\section{Conclusions}

It is possible to roli a multifilamentary composite with copper-nickel matrix to a multifilamentary tape with a factor 3 diameter reduction in one step. The superconducting properties of the wire are maintained. AC field losses in fields parallel to the tape are reduced by about the same factor. The AC curxentcarrying capacity in coil geometry is improved.

\section{Acknowledgement}

The authors wish to thank Mr. J.P.H. Benschop for his contribution to part of the experimental work described in this paper.
References

1. I. Hlásnik, "Prospects of multifilamentary superm conductor AC $50 \mathrm{~Hz}$ applications", Journal de Physique 45, p. C1-459, 1984.

2. H. Riemersma et al., "Application of superconducting technology to power transformers", IEEE Trans. on Power Apparatus and Systems PAS-100, p. 3398, 1981.

3. T. Ogasawara et al., "A low loss NoTi multifilamentary composite for AC use", IEEE Trans. on Magnetics MAG-19, p. 248. 1983.

4. J.L. de Reuver et al., "AC loss contributions of Transport Current and Transverse Field caused by the combined action in a multifilament wire", to be presented at this conference, paper BM-8.

5. H. London, "Alternating current losses in superconductors of the second kind", Phys. Letters $\underline{6}$, p. 162,1963 ,

6. J.H. Murphy, "Experimental investigation of the current distribution characteristics of a superconducting transformer", Advances in Cryogenic Engineering 27, p. 73, 1982.

7. F. van Overbeeke et al., "Investigation of a protection system for transformers with supercunducting windings", presented at the 10th International Cryogenic Engineering Conference, Helsinki, july 31 st - august 3ra 1984 .

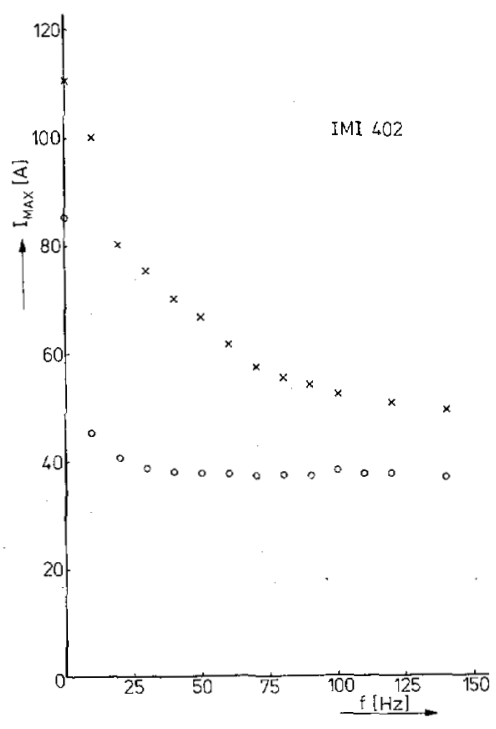

Fig. 3. Maximum current of $402 \mathrm{fil}$. wire as a function of frequency: round wire (0) and rolled wire ( $x$. . 


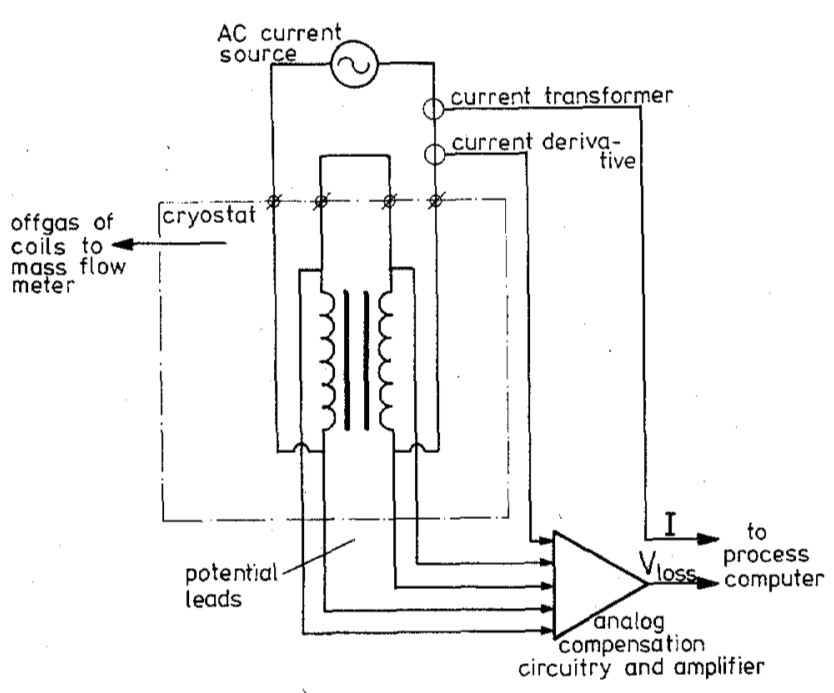

Fig. 4. Loss measurement set-up.

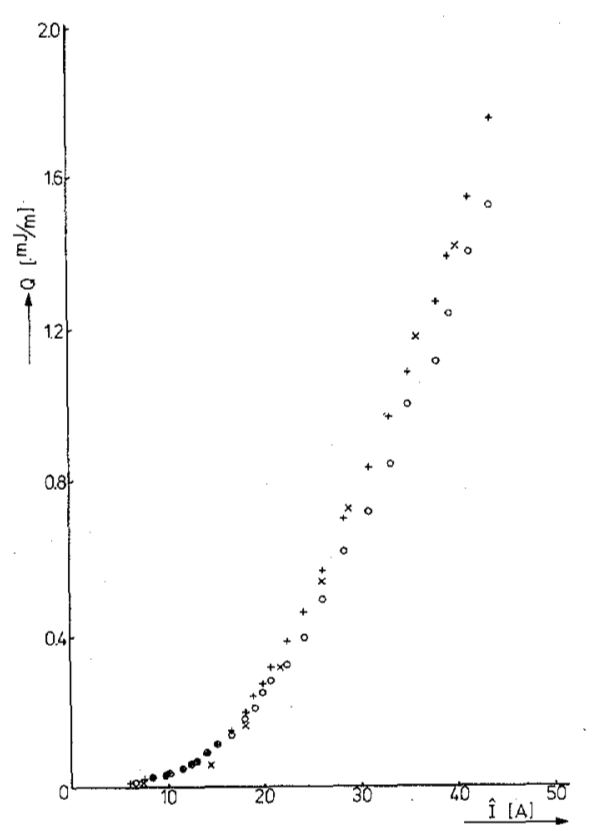

Fig. 5. Losses of transformer windings: electrically measurea $(+)$

calorimetrically measured (0)

calculated from small-coil results $(x)$.

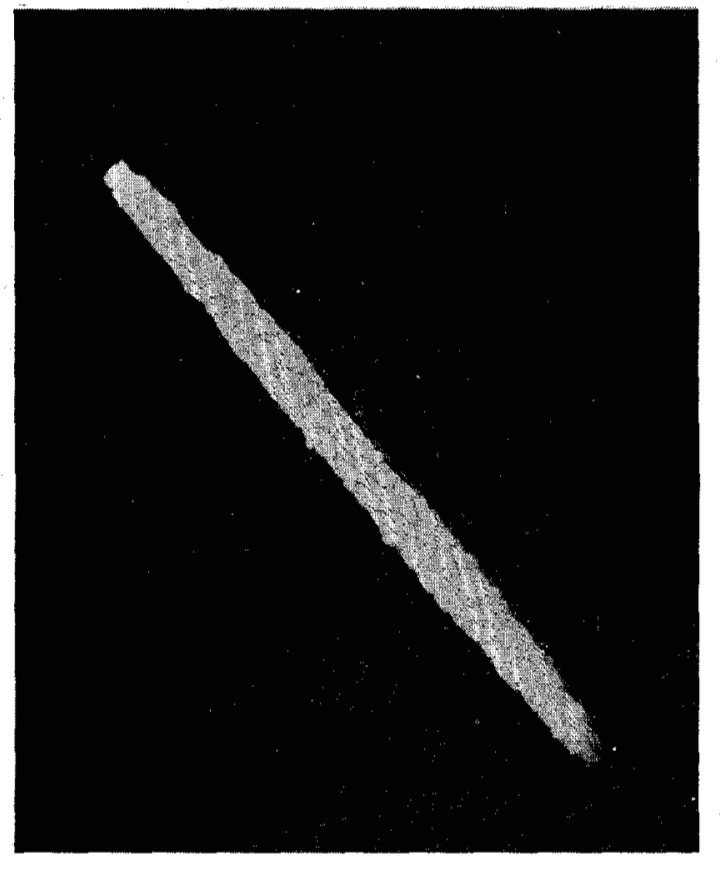

Fig. 6. Photomicrograph of rolled composite.

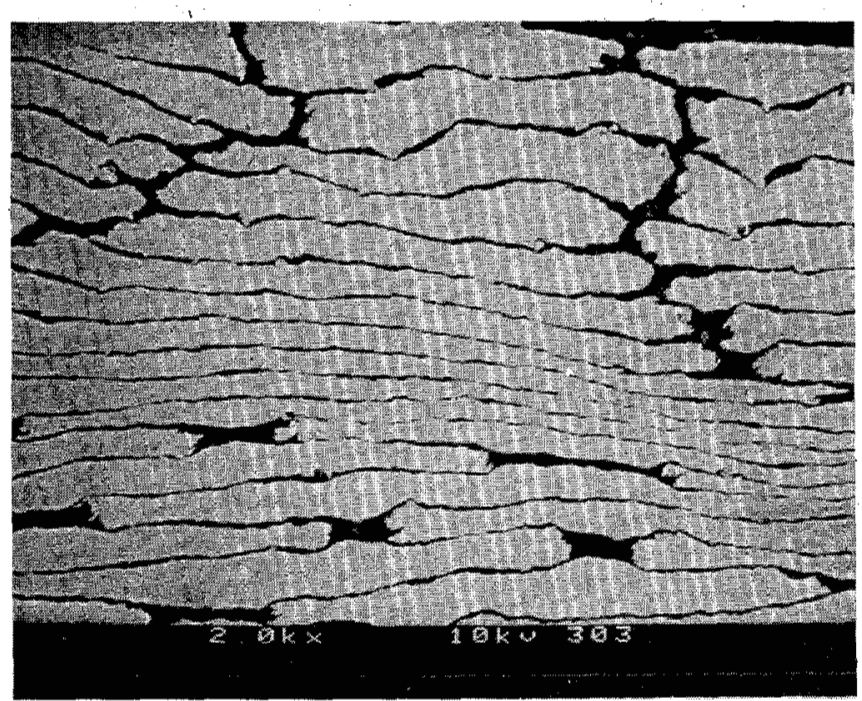

Fig. 7. Detail of fig. 6 . 